Revue internationale P.M.E.

Économie et gestion de la petite et moyenne entreprise

\title{
Pour une lecture politico-institutionnelle du modèle industriel italien et en particulier de la forte présence de PME
}

\section{Ronny Bianchi}

Volume 9, numéro 2, 1996

URI : https://id.erudit.org/iderudit/1008264ar

DOI : https://doi.org/10.7202/1008264ar

Aller au sommaire du numéro

Éditeur(s)

Presses de l’Université du Québec

ISSN

0776-5436 (imprimé)

1918-9699 (numérique)

Découvrir la revue

Citer cette note

Bianchi, R. (1996). Pour une lecture politico-institutionnelle du modèle industriel italien et en particulier de la forte présence de PME. Revue internationale P.M.E., 9(2), 103-123. https://doi.org/10.7202/1008264ar

\section{Résumé de l'article}

Le but de l'article est de mettre en évidence l'importance de la spécificité de la régulation institutionnelle italienne sur le modèle industriel. La dynamique politico-institutionnelle a été à la base de la forte présence de petites et moyennes entreprises (PME) dans le Nord-Est-Centre (NEC) du pays, mais aussi de celle des grandes entreprises (GE) dans la partie nord occidentale. Cette situation a mis aussi en place deux systèmes de production différents : en simplifiant, on retrouve un fordisme hybride dans les GE et la spécialisation flexible dans le NEC. Aujourd'hui, cette distinction est moins marquée et une synergie entre les deux sous-systèmes semble pouvoir exister à la faveur de l'émergence d'un nouveau système de production à l'échelle internationale, dans lequel l'industrie italienne peut s'insérer positivement.
Tous droits réservés @ Presses de l’Université du Québec, 1996
Ce document est protégé par la loi sur le droit d'auteur. L'utilisation des services d'Érudit (y compris la reproduction) est assujettie à sa politique d'utilisation que vous pouvez consulter en ligne.

https://apropos.erudit.org/fr/usagers/politique-dutilisation/ 


\section{Pour une lecture politico-institutionnelle du modèle industriel italien et en particulier de la forte présence de PME}

Ronny BIANCHI

Université de Paris XIII

MOTS CLÉS

Trajectoire historique - Structure institutionnelle - PME

Grandes entreprises - Systèmes de production

Architecture organisationnelle

\section{RÉSUMÉ}

Le but de l'article est de mettre en évidence l'importance de la spécificité de la régulation institutionnelle italienne sur le modèle industriel. La dynamique politico-institutionnelle a été à la base de la forte présence de petites et moyennes entreprises (PME) dans le Nord-Est-Centre (NEC) du pays, mais aussi de celle des grandes entreprises (GE) dans la partie nord occidentale. Cette situation a mis aussi en place deux systèmes de production différents: en simplifiant, on retrouve un fordisme hybride dans les GE et la spécialisation flexible dans le NEC. Aujourd'hui, cette distinction est moins marquée et une synergie entre les deux sous-systèmes semble pouvoir exister à la faveur de l'émergence d'un nouveau système de production à l'échelle internationale, dans lequel l'industrie italienne peut s'insérer positivement.

\section{L'AUTEUR}

Ronny Bianchi est détenteur d'un doctorat en sciences économiques de l'Université de Paris XIII, où il a soutenu une thèse sur le modèle industriel italien. II s'occupe d'économie industrielle et des dynamiques des systèmes économiques. II est chercheur au CREI (Centre de recherche en économie industrielle) de I'Université de Paris XIII. II a récemment publié, en collaboration avec B. Coriat, une étude de cas sur Swatch: Swatch: An European Response to the Japanese Challenge. Adresse : Piazza Collegiata 1, 6500 Bellinzona, Suisse. 


\begin{abstract}
The objective of this article is to show the consequences of the particular institutional structure of the Italian industrial model. The political-institutional dynamic played a very important role for the presence of small and medium factories in the North-East-Centre (NEC) and of big factories in the North-West part of the country. This subdivision has put up, in the same time, two different models of production. To simplify: one model of hybrid fordist in the big factories, and a flexible specialisation model in the NEC. This distinction is today less evident, and synergy between the two models is possible consequently to the emergence of a new production model at the international level. The Italian industrial structure may find a positive place inside this new trajectory.
\end{abstract}

\title{
RESUMEN
}

El objetivo de este articulo es evidenciar la influencia de la particular estructura institucional italiana sobre el modelo industrial. La dinámica politico-institutional ha sido la base para la importante presencia de pequeñas y medianas empresas (PyMEs) en el norte-oriental y central del país (NEC), asi como la presencia de grandes empresas en la parte norte-occidental. Esta subdivisión ha generado paralelamente dos modelos de producción distintos : simplificando, un fordismo híbrido en las grandes empresas y una especialisación flexible en el NEC. Esta diferencia aparece hoy menos evidente y las sinergias entre los dos modelos puede existir come consecuencia de la emergencia de un nuevo modelo de producción sobre el plano internacional, en el interior del cual la industria italiana puede inserirse de manera positiva.

\section{Introduction}

L'espace d'un article étant trop limité pour exposer l'ensemble des problématiques liées au modèle industriel italien, on en proposera les éléments de base ${ }^{1}$ qui, même s'ils sont largement traités par la littérature ${ }^{2}$, restent néanmoins indispensables pour cerner l'aspect central qui nous intéresse : la relation entre la structure politico-institutionnelle et le modèle industriel italien. Il s'agit de vérifier le rôle déterminant de cette relation dans la trajectoire industrielle italienne, partagée entre grandes entreprises (GE) et petites et moyennes

1. Il me semble utile d'informer le lecteur que cet article est la synthèse d'une partie de ma thèse de doctorat, thèse traitant largement des éléments de base mentionnés dans le texte Bianchi (1994).

Je tiens, en outre, à remercier les trois lecteurs anonymes pour leurs remarques et leurs suggestions ainsi qu'Aldo Enrietti avec lequel j'ai pu discuter d'importants passages de ce travail. Toutefois, la responsabilité pour d'éventuelles erreurs m'incombe entièrement.

2. En français voir: Maruani, Reynaud et Romani (1989) et le numéro monographique de la Revue internationale de la PME (1989). 
entreprises (PME). Mon but est de montrer comment l'étude de la régulation institutionnelle de l'Italie est essentielle à la compréhension du modèle économique.

\section{1. Éléments de base du modèle industriel italien}

L'attention des chercheurs pour le modèle industriel italien est centrée principalement sur le rôle des PME à l'intérieur des districts industriels du Nord-Est-Centre (NEC) du pays qui enregistrent-depuis plus de trois décennies - des performances, en termes de compétitivité, difficiles à interpréter avec les instruments traditionnels de l'analyse économique. En outre, plusieurs travaux voient dans le système de production des districts un modèle de rechange au modèle fordiste qui pourrait être adapté à d'autres réalités avec des résultats semblables. Cette hypothèse n'apparaît pas assez convaincante. Il est probable - comme j'essaierai de le démontrer dans cet article-qu'on se trouve en présence d'une réalité plus nuancée issue d'une trajectoire particulière de développement, difficilement exportable et probablement complémentaire au système de production des GE. Il est toutefois nécessaire de préciser que les études sur le système industriel italien, et tout particulièrement sur les districts industriels, ont une grande importance, non seulement dans la compréhension de la réalité du NEC, mais aussi d'un point de vue strictement théorique. Mon objectif est donc de proposer une analyse plutôt complémentaire, par une lecture différente de la réalité productive italienne, sujet, à ma connaissance, insuffisamment traité. Pour ce faire, je procéderai par étapes en commençant par les éléments de base du modèle industriel italien.

\subsection{Une unité politique récente et un développement économique tardif}

Pour comprendre la réalité économique d'aujourd'hui, il faut considérer deux points importants :

1. Dans l'optique européenne, l'unité italienne est très récente : l'État national italien est né en 1860 , après l'unification de toute une série d'États qui avaient une tradition séculaire d'indépendance.

2. Le processus d'industrialisation du pays, selon les caractéristiques fordistes du capitalisme moderne, a débuté plus tard que dans les autres pays industrialisés. À la fin de la Deuxième Guerre mondiale, l'industrie italienne n'était encore que peu développée et pas compétitive. 
Ces deux éléments ont déterminé les particularités du développement économique italien. Alors que dans les autres pays, la grande entreprise industrielle et les structures financières jouaient déjà un rôle central depuis le $\mathrm{XIX}^{\mathrm{e}}$ siècle (Chandler, 1990), l'Italie, sur la route de l'unification, était encore centrée sur un système productif essentiellement agricole et, dans la meilleure des hypothèses, sa structure industrielle était encore fragmentée et partielle.

Jusqu'à la fin du siècle, la structure industrielle du pays demeure centrée sur la petite entreprise traditionnelle, à faible contenu technologique, à gestion familiale et souvent insérée dans un réseau de travail à domicile, sans qualification, emprunté à l'agriculture lors des périodes de faible activité. Ce n'est qu'au début du $\mathrm{xx}^{\mathrm{e}}$ siècle, à la faveur d'une politique protectionniste et d'une intervention directe de soutien de la part de l'État, qu'on assiste à un développement partiel de secteurs industriels modernes comme la mécanique, la chimie et la métallurgie. Parallèlement, une classe dominante, liée à l'ancienne bourgeoisie, se consolide ; mais elle est incapable de dynamiser le processus de développement capitaliste. La structure sociale reste surtout précapitaliste : la consommation est inhibée par une forte imposition fiscale indirecte, tandis que les faibles coûts salariaux sont garantis par des lois répressives (Graziani, 1979 ; Balcet, 1995). La grande entreprise industrielle, située surtout dans le nord-ouest du pays, reste, pour sa part, loin derrière celle des autres pays industrialisés comme les États-Unis, le Royaume-Uni, la France et l'Allemagne, qui ont poursuivi depuis la fin du $\mathrm{XIX}^{\mathrm{e}}$ siècle un processus soutenu d'intégration verticale et de modernisation des grandes entreprises. Malgré une rapide libéralisation économique depuis 1945, par rapport surtout au marché européen ${ }^{3}$, la présence des grands groupes, privés et publics, reste faible ${ }^{4}$, tandis que la grande partie du secteur industriel est composée d'entreprises ayant moins de 500 employés.

\subsection{Le développement industriel conçu selon le modèle dualiste n'a pas donné les résultats espérés}

Les autorités politiques se préfiguraient une industrialisation du pays selon les hypothèses du modèle dualiste qui aurait dû étendre le développement industriel à l'ensemble du pays en partant du Nord-Ouest (Valli, 1993). C'est ainsi que naît, au début du siècle, le Triangle industriel (TI) qui comprend les villes de Turin, Gênes, Milan et qui développe des structures industrielles capitalistes dans la mécanique et la métallurgie (Gualerni, 1994). Là, la bourgeoisie locale,

3. Pour une vision détaillée de l'histoire économique de l'après-guerre jusqu'aux années 1970, voir Graziani (1979), surtout l'introduction.

4. Fiat, le premier groupe industriel italien, n'est que le quinzième en Europe. 
traditionnellement ouverte et en contact avec les autres pays européens, soutient ce développement. L'ensemble de la société se modifie au début du siècle : la bourgeoisie se transforme en classe capitaliste en devenant soit industrielle, soit bancaire. Parallèlement, la force de travail coupe ses liens avec la tradition agricole en devenant une classe ouvrière typique des sociétés industrielles, surtout après la Deuxième Guerre mondiale.

Le Centre et les zones du Nord-Est (NEC) abandonnent progressivement, notamment après 1945, leurs structures agricoles pour passer à une industrialisation poussée, mais centrée sur des petites unités productives gérées selon des critères familiaux et issues de la tradition agricole (Bagnasco, 1988). Les structures sociales et publiques s'orientent peu à peu vers le développement du secteur industriel. La phase extensive de ce processus se situe entre la fin des années 1960 et le début des années 1970, parallèlement à la décentralisation productive de la grande entreprise qui entraîne un passage significatif de maind'œuvre et de connaissances techniques du TI au NEC (Michelsons, 1989).

Le Sud n'a jamais connu de véritable développement industriel. La structure sociale de cette région a toujours été centrée sur un système agricole féodal, géré par la grande propriété foncière, détentrice aussi du pouvoir politique. Contrairement aux agriculteurs du Centre, qui ont progressivement développé une "connaissance d'entrepreneur » en étant propriétaires de leur terre ${ }^{5}$, il n'y avait au Sud qu'une main-d'œuvre agricole sans pouvoir ni connaissances. La réforme agricole de l'après-guerre a permis la formation d'une nouvelle classe de petits propriétaires, tandis que les grands propriétaires se sont tournés vers les investissements immobiliers. De leur côté, les investissements faits par la Cassa del Mezzogiorno ${ }^{6}$ (née en 1950) ont créé une nouvelle classe de petits propriétaires et une petite bourgeoisie d'État fortement liée à la classe politique au pouvoir sur le plan national. Cette classe politique locale était plus intéressée à la distribution des fonds de la Cassa selon des critères politiques qu'à une réelle modernisation de la structure économique et sociale. Les fameuses cattedrali nel deserto, c'est-à-dire les immenses bâtiments qui auraient dû avoir une activité productive et qui n'ont jamais été opérationnels, sont le résultat de la gestion des financements publics par la classe politique locale et nationale.

En réalité, la politique gouvernementale, favorisant le développement industriel (fordiste) dans les régions qui présentaient déjà des conditions

5. Bien que n'étant pas les propriétaires directs de la terre, les agriculteurs du NEC bénéficiaient d'une large autonomie.

6. Cette institution a été chargée de gérer la modernisation du Sud (donc pas seulement agricole) et disposait de capitaux considérables. 
favorables selon les principes du modèle dualiste, n'a pas obtenu les succès espérés. L'idée était que la consolidation de la structure industrielle du TI débouche sur un développement qui s'étendrait à l'ensemble du pays; mais au lieu de cela, l'écart s'est accru entre le Nord et le Sud (Graziani, 1979). Ainsi, le développement du NEC a été davantage la conséquence d'une consolidation de la tradition agricole et artisanale, favorisé ensuite (années 1970) par la décentralisation de la GE et donnant lieu à une structure de production originale.

\subsection{Développement du système fordiste et du modèle de la spécialisation flexible}

Juste après la Seconde Guerre mondiale - coïncidant avec la période du boom économique - l'industrie italienne essaie d'introduire le modèle de production fordiste, en particulier dans les grandes entreprises industrielles du TI. Celles-ci, dans une période de développement de la structure productive, poursuivent l'intégration verticale nécessaire à compléter le cycle de vie du produit. Les marges de croissance sont importantes en raison du faible niveau de l'industrialisation par rapport aux principaux pays développés et d'une demande interne soutenue. Ầ cette première phase d'industrialisation s'ajoute une autre, depuis les années 1970, qui met en difficulté le modèle fordiste, avec des conséquences non négligeables sur la structure productive du pays.

La deuxième phase démarre à la fin des années 1960, avec les difficultés des GE à gérer le climat politique et social (autunno caldo) et avec la nécessité de trouver des structures de production plus petites, afin de tirer parti de la faiblesse du système fiscal et des meilleures conditions de gestion de la maind'œuvre. Ce changement se manifeste dans deux directions : le développement des PME à la périphérie du système industriel traditionnel (TI) et le déplacement de certaines structures productives au sud du pays, grâce aux aides financières distribuées par le gouvernement. Si le déplacement vers le Sud n'a pas enregistré des succès remarquables sur le plan de l'industrialisation, le développement des PME dans le NEC a été de taille et ce, pour au moins trois raisons :

1. La présence, dans les régions du NEC, d'anciennes structures artisanales et de petites entreprises insérées dans un contexte socioéconomique favorable.

2. La disponibilité mentale de la population à développer «l'esprit d'entreprise» dans des petites villes dotées des services nécessaires tels que des réseaux de communication et une formation professionnelle de bon niveau.

3. Par ailleurs, l'intégration verticale des entreprises du TI atteignait son niveau de rupture (présence de désavantages économiques), en raison 
aussi bien de l'augmentation des tensions sociales que de la nécessité de dynamiser (ou de rendre flexible) la structure de l'entreprise. On a donc assisté à une désintégration verticale de la GE associée à une décentralisation de la production vers les PME. Il faut tout de même souligner qu'il ne s'agit pas d'une décentralisation visant une structure productive souple et flexible - comme on a pu le vérifier pendant les années 1980 dans les principaux pays industrialisés - mais d'une stratégie dictée par le climat politique et social. N'empêche que cela a abouti à la création d'une structure de PME très étendue.

$\mathrm{Si}$, dans le TI, le système de production fordiste s'est développé selon une trajectoire hybride (Wolleb, 1986) en raison d'un marché trop petit (le marché italien est relativement fermé sur le plan national) pour permettre une vraie production de masse, dans le NEC, se développe un autre système de production, qui a été défini comme le modèle de la spécialisation flexible (Piore et Sabel, 1984). Bien que susceptible d'être critiqué, ce modèle symbolise bien le système de production de la région qui, même empiriquement, montre sa constance dans le temps (Barca et Magnani, 1989; Bianchi, 1994).

La structuration de deux modèles de production différents, l'un dans la partie occidentale et l'autre dans la partie orientale du pays, est sûrement la conséquence des éléments exposés synthétiquement plus haut et qui ont été amplement mis en évidence dans plusieurs études. Toutefois, il est difficile de comprendre le modèle industriel italien sans prêter attention à la structure politique et institutionnelle qui est à la base d'un régime d'accumulation atypique.

\section{La structure politico-institutionnelle}

La trajectoire historique particulière du pays a influencé le rapport entre les institutions nationales et régionales. À la différence des États fédéralistes, comme les États-Unis et l'Allemagne, en Italie, les régions peuvent avoir des marges de manœuvre importantes dues à la faiblesse et à l'inefficacité des structures nationales. Mais cette relative indépendance des régions n'est pas structurée selon le schéma des États fédéralistes et de plus, elles ne bénéficient pas non plus des avantages d'un État centralisateur comme la France. Les régions italiennes se trouvent donc dans une situation particulière, globalement négative, ne possédant ni l'attrait des structures nationales décentralisées, ni celui des pays fortement centralisés. Il est donc évident que chaque région a développé des trajectoires individuelles dépendant de plusieurs facteurs : avant tout, la volonté des forces politiques régionales de mettre en place les structures nécessaires (ou possibles) au développement économique, qui tiennent compte aussi de l'histoire économique et sociale de la région. 


\subsection{Un système politique aléatoire et instable depuis $\mathbf{1 9 4 5}$}

Partons de la situation actuelle. L'enquête «mains propres » a fortement secoué une grande partie de la classe politique italienne qui a gouverné le pays au cours de l'après-guerre. Cette classe politique empochait régulièrement des «pots-de-vin » pour chaque adjudication de marché public, s'est partagée la plupart des secteurs institutionnels en raison de la force politique des partis et a «occupé » la plupart des entreprises étatiques en les dirigeant surtout selon des critères d'intérêt politique et social, plutôt qu'économique (Friedman, 1989). La conséquence de tout cela a été de consolider une structure publique inefficace : des services non compétitifs, un secteur sanitaire précaire et incapable de faire face aux nécessités quotidiennes de la population, un système fiscal ridicule, une dette publique supérieure au PNB et, surtout, l'absence d'un programme politique à long terme de développement (Nomisma, 1987; Jacobelli, 1989).

La structure politique italienne est sans doute issue d'un bipartisme imparfait. Depuis la fin de la Deuxième Guerre mondiale, deux forces politiques ont polarisé le consensus : la Démocratie chrétienne (DC) au gouvernement, et le Parti communiste italien (PCI/PDS) jamais légitimé à la gestion du pouvoir. $\mathrm{La} \mathrm{DC}$, avec les autres formations politiques du centre et de la gauche modérée, représentait principalement les intérêts de la bourgeoisie et des industriels, ainsi que ceux des petits commerçants et des agriculteurs, surtout dans le Sud, tandis que le PCI regroupait la classe ouvrière et les petits entrepreneurs du centre. Cette situation, potentiellement explosive pendant certaines périodes (par exemple, le terrorisme des années 1970), a permis à la classe politique au pouvoir (DC) de poursuivre une politique populiste du consensus à large rayon d'action et de garder ainsi le pouvoir au détriment de l'efficacité du système économique.

Sur le plan régional, la situation a été différente. Dans les « régions rouges » de l'Émilie-Romagne et de la Toscane, le pouvoir politique a longtemps été géré par le PCI. Le fait d'être la force d'opposition sur le plan national et un parti de responsabilité politique sur le plan régional «pousse » le PCI à pratiquer une politique réaliste (plus que dogmatique) de soutien à l'économie locale, composée pour la plupart d'artisans et de petites entreprises.

En général, l'opposition statique ${ }^{7}$ de longue durée entre deux forces politiques équivalentes (autour de $30 \%$ des suffrages), mais idéologiquement à l'opposé, introduit des dynamiques déformées dans le développement du

7. Dans le sens qu'il n'y a jamais eu un échange de positions, comme cela devrait être le cas dans une démocratie. 


\section{TABLEAU 1}

\section{Pourcentage d'entreprises et d'employés en Italie} et dans trois régions selon la taille (1991)

\begin{tabular}{lcccccccc}
\hline & \multicolumn{2}{c}{ Italie } & \multicolumn{2}{c}{ Piémont } & \multicolumn{2}{c}{ Émilie-Romagne } & \multicolumn{2}{c}{ Toscane } \\
\hline Taille & Entreprises & Employés & Entreprises & Employés & Entreprises & Employés & Entreprises & Employés \\
\hline 1 à 9 & 94 & 45,5 & 90,8 & 38,9 & 93,6 & 48,2 & 94 & 54,8 \\
10 à 19 & 3,7 & 11,2 & 3,9 & 10,7 & 4 & 12,4 & 4,1 & 14,2 \\
20 à 49 & 1,6 & 10,2 & 2,1 & 12,5 & 1,7 & 11,3 & 1,5 & 11,2 \\
50 à 99 & 0,4 & 5,5 & 0,6 & 8,7 & 0,4 & 5,9 & 0,3 & 4,9 \\
100 à 199 & 0,2 & 4,6 & 0,3 & 6,9 & 0,2 & 5,9 & 0,1 & 3,5 \\
200 et + & 0,1 & 23 & 0,2 & 22,4 & 0,1 & 17,1 & - & 11,4 \\
\hline
\end{tabular}

Source : Recensement de l'industrie 1991.

Note : - donnée statistiquement non significative.

pays. C'est ainsi qu'est né le consociativismo, c'est-à-dire cette forme particulière de gestion politique, sociale et économique italienne qui prévoit des zones d'influence pour les acteurs politiques indépendamment du fait qu'ils soient au pouvoir ou à l'opposition.

Globalement, les conséquences de cette situation sur la structure productive, notamment sur la mise en place d'une politique économique efficace ont été lourdes, surtout dans le sud du pays. Au Nord, la force contractuelle des GE et les performances des districts industriels ont permis, pour le moins, de mettre en place une politique structurelle minimale pour une activité ponctuelle, mais insuffisamment organique sur le plan national.

\subsection{Des structures institutionnelles qui font face aux nécessités}

Les formes institutionnelles issues de cette trajectoire politique ont eu un impact décisif sur la dynamique économique du pays. Sur le plan national, elles se sont manifestées à plusieurs niveaux.

1. Les grandes entreprises publiques (ENI, énergie et chimie ; Italsider, sidérurgie ; Alfa Romeo, mécanique) ont eu un rôle positif pendant les années 1960 dans le développement de l'appareil industriel italien, mais ensuite (années 1970), elles ont été utilisées comme amortisseurs des crises des GE privées (Innocenti, Montedison, Sir) et, dans le Midi, comme instrument de gestion des relations politiques et sociales. Cela a contribué à dégrader le rapport État-économie et à mettre en place un marché intérieur protégé qui, par la suite, a limité la confrontation des GE sur les marchés internationaux. 
2. Sur le plan de la politique budgétaire, la dette financée par les titres publics détenus par les Italiens nécessite des taux d'intérêts croissants (nécessaires au refinancement de la dette) qui ont des répercussions sur les investissements privés et qui doivent être compensés par les transferts publics. Toutefois, les titres publics garantissent une rente financière importante et croissante aux ménages depuis le début des années 1980, permettant ainsi de soutenir la demande interne (Bianchi, 1994).

3. L'action politique, d'une part, soutient le développement par des interventions financières (fiscalisation des charges sociales, CIG/ Cassa integrazione guadagni) et politiques (concertation avec les syndicats), mais, d'autre part, elle n'est pas en mesure de doter le pays de structures publiques, économiques et sociales performantes (CER/ IRS, 1989 et 1992).

TABLEAU 2

Intérêts sur la dette, transferts aux ménages et entreprises et formation brute de capital (en milliards de lires et en \%)

\begin{tabular}{lrrrrrr}
\hline Années & PNB & $\begin{array}{r}\text { DETMAR } \\
\text { en \% PNB }\end{array}$ & $\begin{array}{c}\text { ENDETT } \\
\text { en \% PNB }\end{array}$ & INMOTP & $\begin{array}{r}\text { TRMEN } \\
\text { en \% PNB }\end{array}$ & $\begin{array}{r}\text { TRENT } \\
\text { en \% PNB }\end{array}$ \\
\hline 1976 & 174869 & 34,27 & $-9,1$ & 12,11 & & \\
1978 & 253536 & 44,61 & $-9,4$ & 11,60 & & \\
1980 & 387669 & 44,32 & $-9,3$ & 13,27 & 0,5 & 1,41 \\
1982 & 545124 & 50,09 & $-13,5$ & 12,76 & 0,56 & 1,67 \\
1984 & 725760 & 62,31 & $-13,8$ & 12,39 & 0,85 & 1,85 \\
1986 & 899903 & 71,68 & $-12,3$ & 11,19 & 0,98 & 1,64 \\
1988 & 1091837 & 79,31 & -11.5 & 9,84 & 1,08 & 1,70 \\
1990 & 1312066 & 85,49 & $-10,6$ & 10,80 & 1,16 & 1,42 \\
1992 & 1507190 & 92,85 & $-10,9$ & 11,48 & 1,15 & 1,54 \\
1994 & 1638506 & 111,65 & $-9,6$ & 9,17 & & \\
\hline
\end{tabular}

Source: Élaboration à partir des données Banque d'Italie.

Note : DETMAR : dette sur le marché ; ENDETT : endettement annuel ; INMOTP : intérêts moyens sur les titres publics; TRMEN : transfert aux ménages; TRENT : transfert aux entreprises ;

Sur le plan régional, l'évolution des structures institutionnelles s'est orientée vers le soutien du développement économique dans les régions du NEC, spécialement dans «les régions rouges »; en revanche, dans les régions du Sud, les relations politiques entre les forces régionales et nationales ont plutôt freiné le développement. 
L'évolution des formes institutionnelles - rapport salarial, nature de la compétition entre entreprises, rapports État-économie, régime monétaire et international - est le résultat de la solution particulière que les acteurs sociaux ont apportée aux déséquilibres structurels. Par exemple, le pays a surmonté sa faiblesse en R-D en se spécialisant dans les produits traditionnels où la connaissance des techniques et des produits est plus importante que la recherche de base (tableau 3), tandis que l'inefficacité du système fiscal - qui pèse énormément sur la dette - a favorisé, en revanche, le développement des petites unités qui, en effet, ne sont que difficilement contrôlables ${ }^{8}$.

Nous sommes donc en présence d'un dualisme original entre des structures macro-économiques faibles et inefficaces - insuffisamment intéressées dans la mise en place de structures performantes - et des structures méso-économiques et micro-économiques qui bénéficient d'amples marges d'action. Ces marges permettent aux entreprises d'exploiter les faiblesses du système en développant les potentialités productives qui caractérisent les PME.

TABleau 3

Part du marché (en \%) de l'exportation selon les secteurs de Pavitt

\begin{tabular}{lccccc}
\hline & Années & $\begin{array}{c}\text { Secteurs } \\
\text { traditionnels }\end{array}$ & $\begin{array}{c}\text { Secteurs } \\
\text { à économies } \\
\text { d'échelle }\end{array}$ & $\begin{array}{c}\text { Secteurs } \\
\text { spécialisés }\end{array}$ & $\begin{array}{c}\text { Secteurs à haute } \\
\text { technologie }\end{array}$ \\
\hline Italie & 1982 & 12,3 & 4,3 & 6,0 & 2,6 \\
& 1990 & 11,0 & 4,7 & 6,5 & 2,7 \\
Allemagne & 1982 & 6,7 & 13,2 & 16,0 & 8,0 \\
& 1990 & 6,4 & 13,4 & 16,0 & 7,3 \\
France & 1982 & 5,3 & 7,1 & 5,8 & 10,1 \\
& 1990 & 5,5 & 7,1 & 6,1 & 7,4 \\
Royaume-Uni & 1982 & 3,4 & 3,6 & 7,0 & 5,6 \\
& 1990 & 3,2 & 4,5 & 5,9 & 6,2 \\
États-Unis & 1982 & 7,6 & 11,6 & 22,8 & 26,4 \\
& 1990 & 6,9 & 9,8 & 16,9 & 22,2 \\
Japon & 1982 & 5,8 & 15,6 & 9,1 & 12,9 \\
& 1990 & 4,3 & 12,3 & 11,5 & 17,2 \\
\hline
\end{tabular}

Source: Malerba, F. (1992), «Imprese e sistema Italia nella competizione tecnologica internazionale degli anni 90 », Economia e politica industraile, $\mathrm{n}^{\circ} 73$.

8. Évidemment, si le système politique n'est pas en condition de contrôler l'équité du prélèvement fiscal (ou ne le veut pas), toute évasion (très répandue en Italie) permet aux entreprises de bénéficier de marges financières importantes et donc d'avantages compétitifs par rapport aux entreprises des pays où le système fiscal est équitable. 
La particularité des régulations étatico-politiques italiennes a donc introduit des dynamiques qui ont laissé au système des marges d'action originales et difficiles à juger, mais qui finalement ne semblent pas avoir porté préjudice au développement industriel du pays, du moins dans certains secteurs où le pays conserve des avantages compétitifs remarquables. Enfin, le régime d'accumulation, bien qu'étant l'aboutissement de visées contradictoires, souvent en opposition entre elles et divergeant par rapport à celles des autres pays, a été viable dans le passé et le sera probablement aussi dans le futur.

\subsection{La relation capital-management-marché en Italie : une autre particularité}

Deux formes dominantes du capitalisme moderne ont été mises en évidence : l'anglo-saxonne et l'allemande (Chandler, 1990 et Albert, 1993). Dans le modèle anglais, qui trouve aujourd'hui une application optimale aux États-Unis, la séparation entre la propriété du capital et le management de l'entreprise a été le résultat d'un processus historique qui a nettement séparé le rôle de la propriété financière, qui «possède» le capital (actionnariat), de celui de la direction de la firme (manager) qui apporte les connaissances techniques. Dans le modèle allemand, la propriété de l'entreprise est active et joue un rôle important de trait d'union entre le capital institutionnel et le monde du travail. D'un côté, on a des actionnaires alliés au système bancaire; de l'autre, une direction de l'entreprise «alliée » aux forces syndicales. Le modèle n'est pas, comme on pourrait l'imaginer, conflictuel (voir l'exemple de Wolkswagen), mais plutôt coopératif. Le modèle japonais s'approche plus du modèle allemand que du modèle anglais, bien qu'avec des différences importantes. Au Japon, l'interaction entre capital financier et propriété de l'entreprise est très poussée et, dans plusieurs cas, difficilement séparable (Coriat, 1991).

Le modèle italien est doté d'une structure se situant entre le modèle anglais et le modèle allemand, non pas à mi-chemin entre l'un et l'autre, mais contenant les deux formes d'une manière hybride. Dans certains cas, peu nombreux en vérité, la famille propriétaire a évolué vers des formes de séparation entre propriété actionnaire et management, mais dans la plupart des cas, propriété et direction sont entre les mains des mêmes personnes et les rapports entre entreprises et grandes banques, et donc marché actionnaire, n'ont jamais été chaleureux. En Italie ${ }^{9}$, seulement $4,7 \%$ des entreprises sont des sociétés de capital tandis qu'en France elles représentent 14,8\% et en Allemagne 12,9\%.

9. Ces données sont tirées d'une importante étude de la Banque d'Italie sur la propriété et le contrôle des entreprises. Voir en particulier Cannari, Marchese et Pagnini (1994). 
En général, la capitalisation des entreprises en Bourse est très faible (environ $12 \%$ contre $85 \%$ au Royaume-Uni) et la forme la plus répandue de propriété d'entreprises est la familiale (50\% contre $27 \%$ en France et $17 \%$ en Allemagne). Dans les districts aussi, les banques régionales, qui ont un rôle important et presque institutionnel, continuent à opérer comme consultants sans que la séparation entre propriété et capital financier soit remise en question (tandis que celle entre propriété et management n'existe pas).

En Italie, peut-être plus que dans les autres pays, cette structuration du système industriel est le résultat d'une longue tradition historique, culturelle et sociale, décrite par les trajectoires exposées dans les paragraphes précédents ${ }^{10}$. La faible «culture» italienne dans l'organisation industrielle est due à une unité politique récente qui n'a pas su intégrer les spécificités régionales aux originalités institutionnelles et politiques, le tout à l'intérieur d'un marché protégé et technologiquement faible ${ }^{11}$.

L'idée de surmonter les faiblesses apparentes de la structure industrielle italienne par la création de la grande dimension industrielle inspirée du modèle anglo-saxon n'est pas partageable. Les discussions sur la séparation entre propriété de l'entreprise et management, ou sur l'optimisation du capital dans la grande entreprise sont, pour l'Italie, de faux problèmes. La discussion devrait plutôt porter sur le fait que le pays n'a jamais su élaborer de grandes structures industrielles et que les dimensions optimales se sont révélées être la petite et la moyenne entreprise. De plus, l'histoire économique n'a pas démontré, malgré plusieurs études, que le modèle de la grande structure industrielle est le seul praticable. En effet, l'hypothèse contraire est tout aussi valable: plus le pourcentage de capital détenu par le dirigeant est important, plus l'écart par rapport à l'objectif traditionnel de maximisation de la valeur est faible et plus la firme est performante (Charreaux, 1995).

En conclusion de cette deuxième partie, on peut donc observer la présence d'une structure institutionnelle nationale incapable de mettre en place

10. À la faible propension des entreprises à s'adresser au marché de la Bourse, il y a des motifs techniques (McKinsey, 1992), mais aussi des motivations institutionnelles et historiques: le poids des dossiers informatifs demandés par les structures de contrôle; les implications fiscales liées à une transparence accrue dans la gestion du budget de la société et surtout la crainte des entrepreneurs de perdre le contrôle de la gestion de l'entreprise, ce qui est implicite au marché boursier.

11. Le rôle des unités artisanales du Moyen Âge - caractérisées, elles aussi, par une grande capacité d'apprentissage par la pratique et l'utilisation - semble trouver une continuité historique dans les PME qui gardent une forte volonté de contrôle de l'activité de l'entreprise par la propriété du capital. 
une politique industrielle de longue période (R-D, formation, services, système fiscal). Par contre, les entreprises ont essayé de faire face aux nécessités. Dans le TI, la GE, par son rôle de premier plan dans le contexte industriel italien (Fiat, Olivetti, etc.), a toujours bénéficié de l'intervention et du soutien de la classe politique nationale se manifestant par des mesures « au jour le jour» (fiscalisation des charges sociales, CIG, politique monétaire) finalisées à la stabilité politique plus qu'à une réelle volonté de politique industrielle (Giavazzi et Spaventa, 1989). Dans le NEC, les PME sont restées en marge de la politique nationale, mais elles ont pu bénéficier du soutien des structures locales.

Les faiblesses structurelles nationales ont des conséquences sur la spécialisation productive du pays basée (forcément) sur les produits traditionnels et à moyenne technologie ; faiblesses qui ont pu être surmontées, en partie, par de remarquables capacités d'adaptation à l'évolution du marché. Certes, les défis futurs ne sont pas négligeables sur le plan national, et à l'intérieur de l'entreprise aussi, il est nécessaire de développer une culture industrielle plus moderne. La gestion familiale doit pouvoir faire face à d'autres acteurs économiques et être capable de se remettre en question, sans perdre pour autant ses spécificités historiques et productives.

Mais si on considère que de nouveaux principes de production, complémentaires ou substitutifs au fordisme, semblent être en train de s'imposer - par exemple, l'évolution technologique et l'internationalisation des marchés qui mettent au deuxième plan la dimension de l'entreprise par rapport à son organisation - et si on les compare à la trajectoire du développement italien, on s'aperçoit que le pays détient des atouts non négligeables. Il est tout de même utile, avant de répondre à cet argument, de s'arrêter sur la position actuelle du système industriel italien.

\section{Traits communs de la trajectoire du système industriel}

En tenant compte des éléments exposés dans la section 2, on s'aperçoit que le système industriel italien a des racines aussi bien dans le TI que dans le NEC, ce qui peut avoir des répercussions sur son développement futur. Voyons maintenant les traits dominants de la structure industrielle.

Pendant les années 1960 et 1970, les principaux pays industrialisés ont concentré leur système industriel dans les grandes entreprises, tandis que l'Italie poursuivait le renforcement de sa structure centrée sur les PME (Sabel et Zeitlin, 1982). Celles-ci ont gardé leur compétitivité, en adoptant des 
stratégies productives divergentes par rapport aux principes fordistes qui ne conviennent pas à leur dimension ${ }^{12}$. La recherche de flexibilité productive et la qualité des produits ont été, par conséquent, un choix obligé et payant. De plus, les PME des districts se sont tournées, avec succès, vers de nouveaux principes productifs ${ }^{13}$, en introduisant des trajectoires originales tout en respectant une continuité historique, cela bien avant leur application dans d'autres pays, notamment au Japon. Leur principale faiblesse se trouvait dans la structure organisationnelle, en particulier dans la gestion de l'évolution technologique et de l'internationalisation des marchés. Il n'était pas imaginable, non plus, que les PME puissent se doter d'une organisation capable de faire face aux nouvelles nécessités de production. L'intervention des acteurs institutionnels des districts, grâce aussi à une tradition sociale et politique commune, a permis de compenser cette faiblesse par la création de structures de services interactifs et externes à l'entreprise, qui, ainsi, a pu atteindre l'efficacité productive nécessaire (Perulli, 1995). De cette manière, les PME des districts ont accédé à une dimension productive comparable à celle de la GE, bien qu'organisée différemment. (Bianchi, 1994)

De leur côté, les GE ont essayé avec un certain succès d'introduire, depuis la fin de la Deuxième Guerre mondiale, les principes de production fordiste qui, toutefois, entraient en conflit avec les limites de la structure sociale et industrielle du pays. Ce n'est qu'avec la restructuration des années 1980, selon le modèle de la production diversifiée de qualité (Streeck, 1991) ou de la production de masse flexible (Boyer et Coriat, 1989), que la GE semble trouver un emplacement «plus naturel » à l'intérieur du système industriel italien. La décentralisation de la GE des années 1970 avait pour objectif d'échapper aux contrôles institutionnels - plus faibles dans les PME (Regini, 1995) - , ce qui a permis dans la décennie suivante de jeter les bases d'une organisation plus souple et plus en mesure de réagir aux changements de la demande internationale et des systèmes de production.

En conclusion, si on examine la trajectoire du développement italien dans sa globalité, la séparation entre GE du TI et PME du NEC apparaît

12. Le modèle de la spécialisation flexible proposé par Piore et Sabel (1984) s'adapte bien à cette réalité.

13. Les principes productifs suivants caractérisent une grande partie des PME: intégration entre nouvelles technologies, production et vente; intégration producteurs-utilisateurs et diversification; versatilité de la production en réponse à la demande ; coordination horizontale par réseaux de partenariat ; compétence et attachement des salariés à l'entreprise ; compromis à long terme : compétence et loyauté contre emploi et salaires élevés (voir aussi la suite du texte). 
moins nette ${ }^{14}$. Certes, les réalités productives sont différentes, mais les deux systèmes ont été obligés de se développer (ou de se débrouiller) à l'intérieur de la même organisation institutionnelle nationale qui influence les stratégies opérationnelles des structures productives (GE, districts, PME). On serait plutôt en présence d'une complémentarité entre les deux «sous-systèmes » (TI et NEC) dictée par la trajectoire historique, politique, institutionnelle et culturelle du pays, bien que, globalement, le climat reste plus favorable au développement des PME.

Le fait que les deux systèmes soient finalement assez perméables permettant aussi le passage de la dimension de PME à celle de GE (et non seulement l'inverse), grâce aux réseaux industriels et à la présence de groupes d'intérêts - est prouvé par l'évolution d'entreprises du type Benetton. Celles-ci ont débuté comme petites entreprises, en marge des districts industriels classiques et en quelques années -20 pour Benetton - elles ont atteint la taille, en chiffre d'affaires, des GE, tout en gardant une structure de production particulièrement flexible avec un important réseau de sous-traitants. Elles sont gérées avec des technologies avancées, aussi bien dans la gestion des informations que dans les stratégies productives (système de franchise). Dans ce cas, on peut même dire qu'il s'agit d'un leader - l'entrepreneur innovateur de Schumpeter - qui utilise au mieux les possibilités technologiques ${ }^{15}$.

\section{Conclusions : convertir les désavantages en avantages}

Finalement, la force d'un système de production national réside dans sa capacité de se gérer à l'intérieur de sa structure institutionnelle et politique. Or, si on considère l'évolution réelle des systèmes de production (Boyer et Freyssenet, 1995) et si on applique cette définition à l'Italie, celle-ci est sûrement en mesure de transformer en avantages compétitifs non négligeables les aspects négatifs du système de production fordiste qui représentaient des désavantages. En partant des principales caractéristiques du modèle italien, voyons comment peut s'effectuer cette transformation.

14. Une étude récente (Barca et al., 1994) a mis en évidence comment la forme juridique la plus répandue est celle du " groupe », même parmi les PME, bien que la signification du terme soit différente pour les petites entreprises - où on a plutôt un groupe familial - de celles des grandes où le contrôle financier est plus structuré.

15. « On trouve donc un réseau télématique mondial pour les commandes, l'automation du magasin central et la planification de l'adjudication des contrats, de façon à produire le maximum d'efficacité de production et la plus grande adéquation avec les différents marchés. » (Enrietti, 1989) 
Éléments de la trajectoire politico-institutionnelle italienne... :

- histoire politique récente et difficulté d'uniformisation des régions. L'Italie serait à traiter comme une configuration de réalités subnationales qui présentent une cohérence entre économie et institutions (Bagnasco, 1988);

- présence d'une interconnexion entre régulation institutionnelle faible - sur les plans macro-économique et national -et régulation volontariste et efficace mais instable sur les plans micro-économique et régional (Regini, 1995);

- régions - surtout du NEC - capables de se structurer en exploitant les faiblesses nationales et en se dotant de formes institutionnelles (régionales) compétitives; un rapport salarial positif établi par des conventions sociales tacites, des formes de concurrence basées sur le rapport compétition / coopération et une insertion internationale croissante dans les secteurs de spécialisation;

... et éléments de la trajectoire industrielle :

- la spécialisation industrielle du pays est centrée sur les secteurs traditionnels et sur ceux tirés par la demande (scale intensive). De plus, le marché national est relativement fermé, ce qui empêche, jusqu'à la fin des années 1980, une confrontation/compétition des GE sur les marchés internationaux (par exemple, le secteur de l'automobile). Souvent, le taux de change a été utilisé pour relancer les exportations;

- sur le plan national, les structures de R-D sont faibles et empêchent un passage aux nouvelles technologies, bien que les investissements dans les hautes technologies n'aient pas nécessairement de répercussions directes sur la productivité industrielle (Napolitano, 1991) (Amable et El Mouhoub, 1990). Des faiblesses sont aussi présentes dans la formation et dans les services;

- dans le NEC, les PME ont montré des capacités remarquables de learning by doing et by using, ce qui leur permet d'être compétitives sur le marché mondial et dans leurs secteurs de spécialisation. Leur avantage réside dans la capacité d'innover dans les secteurs traditionnels en «exploitant» les informations en provenance des marchés et des autres secteurs (learning in action) (Di Bernardo et Rullani, 1994);

- malgré de réelles difficultés institutionnelles et structurelles, les entreprises italiennes (manufactures) ont été, dans les années 1980, les seules parmi celles du G7 à afficher un taux de productivité croissant par rapport au Japon (Bianchi, 1994). 
Or, si on examine les principes qui semblent s'imposer comme les éléments de base du nouveau système de production ${ }^{16}$, on entrevoit la possibilité d'une évolution dynamique de la trajectoire industrielle italienne. La transformation du modèle fordiste (ou de sa phase de crise), depuis les années 1970, a permis la mise en place de différents itinéraires de production qui trouvent en Italie un emplacement particulier :

1. Nous avons d'abord le changement du rapport salarial. Les tensions dans le marché du travail et dans l'organisation de la production ont réduit les bienfaits de la division du travail et de la grande échelle de production. Les GE ont donc essayé, dans les années 1980, de nouvelles formes de gestion du rapport salarial, sans parvenir à des résultats éclatants ; toutefois, les contingences les ont obligées à poursuivre dans de nouvelles voies. Mais c'est surtout dans les PME (nées aussi de la décentralisation de GE) qu'un nouveau modèle est apparu, basé sur le rôle accru de la main-d'œuvre à l'intérieur de l'entreprise.

2. L'extension de la spécialisation et de la diversification de la demande nationale et internationale a eu des répercussions sur la demande des biens de consommation et d'investissement. La fabrication de produits différenciés, souvent en série limitée et surtout dans les produits traditionnels dans lesquels se spécialisent les PME italiennes, a favorisé l'entrée sur le marché de nouvelles entreprises et la réorganisation de celles qui s'y trouvaient déjà.

3. En outre, la flexibilité du capital s'est accrue à la suite des progrès techniques. Les nouvelles technologies permettent la flexibilisation de la production, la réduction des phases de production et de la taille minimale efficace des installations. Les phases du travail les plus pénibles peuvent être accomplies par les machines. De plus, les nouvelles techniques permettent de réduire le capital fixe. Le progrès technologique et la diffusion de l'innovation réduisent l'échelle de production et, par conséquent, le rapport entre taille de l'entreprise et capacité d'innovation.

4. En général, la production standardisée est de moins en moins intéressante pour les pays industrialisés, qui ont à faire face à une concurrence croissante des pays en voie d'industrialisation où les coûts du travail sont meilleur marché.

Or, sans entrer dans les détails en ce qui a trait aux principes du nouveau système de production ${ }^{16}$, les avantages par rapport aux concurrents sont de

16. Pour en savoir davantage sur les aspects théoriques et leurs applications aux principaux pays industrialisés, voir Boyer (1992 et 1995) et pour l'Italie, Bianchi (1994) 
taille. Par exemple, l'architecture organisationnelle est déterminante pour le développement technologique - à l'intérieur du secteur de spécialisation-dans le sens que l'innovation dépend de trois phases : l'invention, sa mise en œuvre et son débouché dans un produit ou service final (Ménard, 1995). En d'autres mots, la capacité de comprendre l'évolution de la demande, d'adapter le système de production à cette évolution et d'être présent sur les marchés au bon moment avec le bon produit est de plus en plus importante, surtout pour les entreprises des pays industrialisés qui doivent être concurrentielles au regard de la qualité, de la flexibilité et de l'adaptation du produit, plus qu'à celui des prix de production qui sont désormais les atouts des pays en voie de développement. Cette situation est l'un des éléments qui font la force des PME du NEC et particulièrement des districts, qui peuvent compter sur une maind'œuvre qualifiée et motivée, sur une connaissance des marchés très grande et surtout sur une flexibilité générale remarquable du système de production. Mais les GE aussi, bien que plus lentement et avec des difficultés majeures, semblent aller dans cette direction, sans compter les entreprises « hybrides » type Benetton ou Stefannel qui sont à l'avant-garde sur le plan mondial.

\section{Conclusion}

Pour conclure, il semble que les faiblesses politiques et institutionnelles aient permis de développer des stratégies de production flexibles qui, a posteriori, se révèlent en accord avec l'évolution en acte, permettant aux entreprises «d'exploiter » leur trajectoire de développement. Les entreprises «postfordistes » ont la possibilité de choisir entre plusieurs solutions aussi bien d'organisation que de marché, mais il semble surtout que chaque milieu institutionnel puisse favoriser certaines stratégies d'organisation et de marché plutôt que d'autres, en donnant aux entreprises, qui sont en mesure de s'adapter, des avantages par rapport aux concurrents des autres pays où la structure institutionnelle a développé divers avantages ou désavantages (Hollingsworth, Schmitter et Streeck, 1994). «Ce sont les entreprises qui exploitent au mieux ce que les institutions offrent - ou qui s'organisent de façon à minimiser les effets des défauts-qui atteignent les meilleures performances et qui donc se reproduisent le mieux à l'intérieur d'un milieu institutionnel donné. » (Regini, 1995, p. 9) Et sur ce point, les PME italiennes, surtout si elles ont la possibilité d'agir à l'intérieur d'un district industriel, montrent des capacités d'adaptation remarquables. Pour les GE, la situation est moins positive à cause de leur structure, forcément plus rigide, mais elles aussi ont montré des capacités d'adaptation au milieu institutionnel même si elles ont adopté des stratégies différentes de celles auxquelles ont recouru les PME. 


\section{Bibliographie}

Amable, B. et M. EL Mounoub (1990), «Changement technique et compétitivité internationale : une comparaison des six grands pays industriels », Revue d'économie industrielle, $\mathrm{n}^{\circ} 54$.

Albert, M. (1993), Capitalismo contro capitalismo, Bologna, Il Mulino.

Bagnasco, A. (1988), La costruzione sociale del mercato, Bologna, Il Mulino.

BALCET, G. (1995), L'économie de l'Italie, Paris, La Découverte.

Barca, F., M. Bianco, L. Cannari, R. Cesari, C. Gola, G. Manotta, G. Salvo et L.F. SIGNORINI (1994), Assetti proprietari e mercato delle imprese, Bologna, Il Mulino.

BARCA, F. et M. MAGNANI (1989), L'industria tra capitale e lavoro, Bologna, Il Mulino

BIANCHI, R. (1994), Le modèle industriel italien : réflexion théorique et historique à la lumière des années 80 , Thèse de doctorat, Paris, Université Paris XIII.

BIANCHI, R. et B. CORIAT (1995), «Swatch : a European response to the Japanese challenge », dans L.E. Andersen, B. Coriat, F. den Hertog et R. Kaplinsky, Europe's Next Step. Innovation, Competition and Employment, Ilford, Franck Cass.

BOYER, R. et B. CORIAT, (1989), « De la flexibilité technique à la stabilisation macroéconomique. Un essai d'analyse », dans P. Cohendet et P. Llerena, Flexibilité, information et décision, Paris, Economica.

BOYER, R. et M. FREYSSENET (1995), L'émergence de nouveaux modèles industriels, Paris, GERPISA, $2^{\mathrm{e}}$ version provisoire.

CANNARI, L., G. MARChese et M. PAgANINI (1994), «Forma giuridica, quotazione e struttura proprietaria delle imprese italiane : prime evidenze empiriche», dans Il mercato delle proprietà e del controllo delle imprese : aspetti teorici e istituzionali », Roma, Banca d'Italia.

CER / IRS (1992), Una politica industriale per la nuova legislatura, Quinto rapporto, Bologna, Il Mulino.

CER / IRS (1989), Mercato e politica industriale. Terzo rapporto, Bologna, Il Mulino

ChAndler, A. (1990), Scale and Scope: The Dynamics of Industrial Capitalism, Cambridge, London, Belknap.

Charreaux, G. (1995), «Mode de contrôle des dirigeants et performances des firmes ", Revue d'économie industrielle, hors-série, p. 135.

Coriat, B. (1991), Penser à l'envers, Paris, Bourgois.

Di BERNARDO, B. et E. RULLANI (1994), « Apprendimento ed evoluzione nelle teorie dell'impresa », Stato e Mercato, $\mathrm{n}^{0} 41$

ENRIETTI, A. (1989), «De la petite entreprise au groupe », Revue Internationale PME, vol. $2, \mathrm{n}^{\text {os }} 2-3$. 
Friedman, A. (1989), Ce la farà il capitalismo italiano?, Milano, Longanesi.

GiavazzI, F. et L. SPAVENTA (1989), «Gli effetti reali dell'inflazione e della disinflazione », Rivista di politica economica, luglio - agosto.

GuAlerni, G. (1994), Storia dell'Italia industriale, Milano, Etas Libri.

GrazIANI, A. (sous la dir. de) (1979), L'economia italiana dal 1945 a oggi, Bologna, Il Mulino.

Hollingsworth, R., P. Schmitter et W. Streeck (1994), Governing Capitalist Economies. Performance and Control of Economic Sectors, New York and Oxford, Oxford University Press.

JACOBELli, J. (sous la dir. de) (1989), Dove va l'economia italiana ?, Roma, Laterza.

MALERBA, F. (1992), «Imprese e sistema Italia nella competizione tecnologica internazionale degli anni $90 »$, Economia e politica industraile, $\mathrm{n}^{0} 73$.

MÉNARD, C. (1995), «La nature de l'innovation organisationnelle : éléments de réflexion ", Revue d'économie industrielle, hors-série, p. 173-188.

MCKINSEY (1992), La Borsa italiana : un progetto paese per un progetto positivo, ronéotypée.

MiCHELSONS, A. (1989), «Structures sociales et aspects institutionnels des systèmes d'industrialisation diffuse de l'Italie du Centre», Revue Internationale PME, vol. $2, \mathrm{n}^{\text {os }} 2-3$.

Maruani, M., E. Reynaud et C. Romani (1989), La flexibilité en Italie, Paris, Syros Alternatives.

NAPOLITANO, G. (1991), «Industrial research and sources of innovation », Research Policy, vol. 20, n⿳⺈ 2 .

Nomisma (1987), La produttività in Italia, Milano, Il Sole 24 Ore.

Perulli, P. (1995), «Stato, regioni, economie di rete», Satato e Mercato, no 44.

Piore, M. et C. SABEL (1984), The Second Industrial Divide, New York, Basic Books.

REGINI, M. (1995), « La varietà italiana di capitalismi. Istituzioni sociali e struttura produttiva negli anni ottanta », Stato e Mercato, $\mathrm{n}^{\circ} 43$.

SABEL, C. et J. ZEITLIN (1982), «Alternative storiche alla produzione di massa », Stato e Mercato, $\mathrm{n}^{0} 5$.

STREECK, W. (1991), «On the institutional conditions of diversified quality production », dans Matzer et Streeck (sous la dir. de), Beyond Keynesianism: The Socio-Economics of Production and Full Employment, Aldershot, Edward Elgar.

VAlli ,V. (1993), Politica economica. Teoria e poltica dello sviluppo. Il caso italiano, Roma, La Nuova Italia Scientifica, p. 254-259.

Wolleb, E. (1986), « D'une institutionnalisation tardive au contournement. L'Italie», dans R. Boyer, La flexibilité du travail en Europe, Paris, La Découverte. 